\title{
Percepción acerca del servicio de las agencias de viajes de Playa del Carmen
}

Irma Guadarrama Gómez Universidad Tecnológica de la Riviera Maya

\section{Resumen}

La presente investigación es de carácter cuantitativo exploratorio, considera un muestreo no probabilístico a conveniencia y se utiliza el modelo Servqual para calcular las brechas de insatisfacción en las cinco dimensiones que lo conforman, con el propósito de identificar el grado de calidad del servicio que ofrecen las agencias de viajes en Playa del Carmen. En este modelo se toman como base las expectativas y percepciones de los clientes. La primera parte de esta investigación contempla la adecuación de la herramienta y su aplicación; en la siguiente se analiza la información recabada mediante la codificación e interpretación de datos. Se concluye que existe un problema importante que afecta al sector que representan las agencias de viajes de este destino, que no permite satisfacer las expectativas de sus clientes, por lo que es necesario proponer estrategias que conlleven a elevar la calidad y profesionalización del recurso humano en el área de servicio.

\section{Palabras clave}

Agencia de viajes, calidad en el servicio, expectativas, percepción, modelo Servqual. 


\title{
Perception of the Travel Agencies service in Playa del Carmen, Quintana Roo, Mexico
}

\author{
Irma Guadarrama Gómez \\ Universidad Tecnológica de la Riviera Maya
}

\begin{abstract}
Abstrac
This research is quantitative exploratory sampling considering probalistic not to convenience and using the Servqual model to calculate dissatisfaction gaps within the five dimensions that comprise it, in order to identify the degree of quality of service offered travel agencies in Playa del Carmen, based on the expectations and perceptions of current customers. The first part of this research, includes the adaptation of the tool and the application of it. In the next step we analyze the information gathered through the coding and interpretation of data. These data showed that there is a major problem affecting the sector representing travel agencies such assignments, service to be assessed with a degree of deficiency that cannot satisfy the expectations of its customers, so it is necessary to propose appropriate strategies that lead to improve the quality and professionalism of human resources in the service area.
\end{abstract}

\section{KEY WORDS}

Travel agency, quality service, expectations, perceptions, Servqual Model. 


\section{Introducción}

La medición y aseguramiento de la calidad en el servicio ayuda a dirigir esfuerzos para satisfacer necesidades de mercados cada día más exigentes y conocedores de las diferentes características que implica un servicio de excelencia. Por lo anterior, es importante que las empresas de servicios ofrezcan un nivel de calidad óptimo, considerando la calidad total se toma como una estrategia que permite consolidar a las empresas dentro de un mercado competitivo y globalizado.

La presente investigación surge por el interés de identificar el grado de calidad del servicio que ofrecen las agencias de viajes en Playa del Carmen y de generar información que contribuya a mejorar la competitividad de estas empresas; las cuales participan en la promoción, creación y comercialización de los diferentes productos turísticos, que a su vez están integrados por los servicios que ofrecen otras empresas del sector. Por lo que profesionalización y la calidad serán factores decisivos para cumplir con las expectativas y necesidades del cliente, beneficiando con ello tanto al consumidor como a las diferentes empresas relacionadas en el proceso y, por lo tanto, esto también impactará en el posicionamiento del propio destino.

Para medir la calidad en el servicio, diferentes investigadores han desarrollado modelos que permiten identificar el grado de calidad que se percibe. Uno de estos modelos es el propuesto por Parasuraman, Zeithaml y Berry en 1985, conocido como modelo Servqual, herramienta que mide la calidad en el servicio mediante la diferencia entre las percepciones y las expectativas de los clientes.

Este trabajo tiene el objetivo de analizar la percepción del mercado sobre el servicio que proporcionan las agencias de viajes de Playa del Carmen. Se exponen los antecedentes más representativos del entorno en el que desarrolla el tema de estudio, considerando también el diseño metodológico, la exposición de los resultados así como la conclusión del trabajo. 


\section{Antecedentes}

Las agencias de viajes han sido un elemento esencial en la elaboración y comercialización de productos turísticos, se objetivo es satisfacer las necesidades básicas de seguridad y comodidad que requiere el turista o cliente durante su viaje o incluso antes. En la actualidad, las necesidades del cliente son tan variadas y complejas que las agencias de viajes se ven obligadas a especializarse para alcanzar los estándares de calidad mundial.

La evolución de la actividad turística ha llevado a que la organización y operación de las agencias de viajes estén en una constante transición, al grado de ir incorporando en su oferta diferentes tipos de servicios que facilitan el traslado y permanencia de los turistas.

Chan (2005: 63) afirma que "a nivel mundial hay aproximadamente 30000 agencias de las cuales según la omt el 70\% está localizado en Europa, el 14\% en Norteamérica, el 8\% en Asia y el Pacífico, el 4\% en América Latina y el $4 \%$ en África y Medio Oriente.” Esta referencia muestra el impacto y alcance que tienen estas empresas en el sector turístico a nivel mundial; siendo las agencias de viajes europeas las que predominan en el sector, lo cual históricamente siempre ha reflejando el liderazgo de algunos países europeos dentro del ámbito turístico, principalmente España, Inglaterra y Alemania.

Entre las tendencias que siguen las agencias de viajes para estar dentro de la competencia y tener una mayor penetración en el mercado está la aplicación de las tecnologías de la información, usadas principalmente para estar en contacto directo con los clientes o posibles consumidores de cualquier lugar del mundo y en cualquier momento, mediante el uso de Internet. Lo anterior les ha permitido difundir sus productos con una mayor cobertura y al mismo tiempo identificar las tendencias o necesidades del mercado.

Los mercados turísticos tienen gran importancia dentro de la dinámica de productividad y posicionamiento de las empresas, por lo que identificar sus características y necesidades permiten que sean compensadas mediante el consumo de los diferentes productos turísticos que se ofertan. El mercado en sí, se puede clasificar desde diferentes enfoques, sin embargo, considerando el tema 
de estudio que se aborda, el enfoque se tomará desde el punto de vista de la demanda, de la cual se deriva una subclasificación: mercado actual y mercado potencial.

Rivera y López-Rua (2007: 69) identifican al mercado actual como "el que en un momento preciso demanda de un producto / servicio determinado". Mientras que el mercado potencial es "el número máximo de compradores al cual se puede dirigir la oferta comercial de la empresa". Por lo que se puede concluir que el mercado actual está reconocido por los clientes que ya consumieron o están consumiendo un producto o servicio, mientras que el mercado potencial lo conforman todos los posibles clientes que todavía no consumen el producto o servicio, pero que lo pueden hacer en un determinado momento. Para ambas clasificaciones las empresas aplican diferentes tipos de estrategias, con la finalidad de captar al mercado potencial y propiciar la fidelización del mercado actual, que es cuando se genera un vínculo importante entre la empresa y el cliente.

Cualquier empresa o institución que desee estar dentro del mercado con una ventaja competitiva tiene el compromiso de ofrecer un servicio de calidad que cumpla o sobrepase las expectativas de los clientes. Para poder cumplir con ese objetivo, las empresas, además de ofertar un producto que satisfaga necesidades, deben de contar con capital humano que tenga las habilidades y capacidades pertinentes y les permita identificar y satisfacer las expectativas de los clientes; desarrollando así una cultura de servicio en beneficio de los usuarios, conocidos también como clientes internos o externos.

Juran (1996: 9) plantea varias definiciones sobre el término de calidad, bajo un enfoque basado en las características del producto, en este sentido "a los ojos de los clientes cuando mejor sean las características de un producto, mayor será la calidad”. El otro enfoque habla de ausencia de deficiencias: "a los ojos de los clientes, cuantas menos deficiencias tenga, mejor será la calidad”.

Las empresas comprometidas en lograr la satisfacción del cliente y tener un mejor posicionamiento en el mercado buscan contar con productos y servicios que tengan altos estándares de calidad, lo que implica para éstas aplicar métodos 
que permitan medir el grado de cumplimiento en relación con las necesidades de los consumidores, considerando además sus expectativas y percepciones.

Se han desarrollado varios métodos y técnicas de tipo cuantitativo y cualitativo para evaluar la calidad en el servicio, y dentro de estos métodos se encuentran la aplicación de herramientas en la recolección de datos para llevar a cabo la investigación; tales como entrevistas, cuestionarios, listas de cotejo o bien el registro de observación documental, entre otros. Definir cuál o cuáles serán las herramientas correctas depende del tipo de investigación, así como la naturaleza de las variables; pero algo que las debe de caracterizar es su validez y confiabilidad hacia el cumplimiento del objetivo de la investigación.

Una herramienta desarrollada desde 1985 por Parasuraman, Zeithaml y Berry es el modelo Servqual, también conocido como pzB por las iniciales de sus creadores, que permite medir la calidad en el servicio mediante la diferencia entre las percepciones y las expectativas de los clientes. Se evalúa la calidad del servicio desde la perspectiva del cliente. Si el valor de las percepciones iguala o supera el de las expectativas, el servicio es considerado de buena calidad, mientras que si el valor de las percepciones es inferior al de las expectativas se dice que el servicio presenta deficiencias de calidad (González, Chamorro y Rubio, 2007).

Dicho modelo identifica cinco dimensiones para evaluar el grado de cumplimiento en el servicio, estas dimensiones son: elementos tangibles, que evalúa la apariencia de las instalaciones físicas, equipos, personal y materiales de comunicación; fiabilidad, cuyas preguntas están orientadas a conocer la habilidad para ejecutar el servicio prometido de forma fiable y cuidadosa; $c a$ pacidad de respuesta, para conocer la disposición y voluntad de los empleados para ayudar al cliente y proporcionar el servicio; seguridad, cuyos ítems están orientados a identificar el grado de conocimiento y atención mostrados por los empleados y sus habilidades para inspirar credibilidad y confianza; la última dimensión esta relacionada con la empatía, que es atención individualizada que ofrecen las empresas a los consumidores.

Tomando en cuenta que el objeto de esta investigación se sitúa en Playa del Carmen es importante destacar que esta ciudad ha tenido un crecimiento y desarrollo económico importante, donde varios factores han influido para su 
transición, de ser un pueblo de pescadores a un destino con potencial turístico reconocido a nivel mundial.

Con un desarrollo muy lento, esta ciudad empieza de destacarse a partir de los años sesenta, con una actividad económica más productiva derivada del progresivo crecimiento de la población que llegaba del interior de la república, en particular de los estados de Yucatán y Campeche, por su colindancia con Quintana Roo.

Por lo que se puede decir que Playa del Carmen es un destino relativamente joven, y de los más reconocidos de la Riviera Maya. Dentro de los datos estadísticos generados por la Secretaría de Turismo de Quintana Roo, Sedetur y el INEGI, entre otras organizaciones, se ha podido cuantificar la siguiente información sobre el destino:

- $\quad 161$ hoteles con 5785 cuartos

- Según la Organización Mundial de Turismo, por cada cuarto de hotel que se construye en esta zona, se generan 1.5 empleos directos y 2.5 indirectos, lo cual beneficia a cuatro nuevas familias por cada habitación edificada.

- La estadía promedio de los huéspedes es de 6.7 días. Registrando 3.2 días para el turismo nacional y 6.9 días para el internacional.

- La tarifa actual promedio es de \$2 465.51 pesos.

- De 532164 turistas extranjeros que visitan la Riviera Maya que concentra a Playa del Carmen se sabe que $42.75 \%$ provienen de los Estados Unidos, Canadá representa 30.19\%, Europa $24.11 \%$, Sudamérica $2.39 \%$ y el resto del mundo $0.56 \%$.

- La estacionalidad de los visitantes nacionales es principalmente en verano, durante los meses de julio y agosto, a diferencia de los extranjeros que presentan su mayor afluencia en el mes de marzo.

- En su entorno natural y cultural se puede encontrar flora y fauna típica de la región así como una gran cantidad de cenotes, ríos subterráneos, playas y una gran variedad de manifestaciones de la cultura maya. 
- Sus principales vías de acceso a esta ciudad son: la Carretera Federal 180; dos puertos marítimos de enlace con el destino turístico de Cozumel y un aeropuerto de corto alcance. Sin embargo a $70 \mathrm{~km}$, en la ciudad de Cancún se cuenta con el segundo aeropuerto internacional de largo alcance más importante del país operado por Aeropuertos del Sureste de México (Asur), cuya afluencia turística promedio es de 3375000 pasajeros, según el Sistema Integral de Operación Migratoria, 2011-2012, lo cual beneficia al destino, considerando que parte de esta afluencia llega a Playa del Carmen.

- En el último censo presentado por el INEGI, realizado al 31 de diciembre de 2009, en el municipio de Solidaridad hay 17 agencias de viajes, aunque se estima que operan más empresas de este tipo, de las cuales sólo Best Day y Olympus Tour son las únicas que están afiliadas a la Asociación Mexicana de Agencias de Viajes (AmAv, Cancún), según información proporcionada por la misma asociación durante la presente investigación.

La planta turística de Playa del Carmen la conforman una gran variedad de empresas de diferentes categorías y ramos, que ofrecen el servicio al turista durante su estancia en el destino; entre éstas figuran diferentes agencias de viajes: Best Day, Olympus Tours, Viajes x el Mundo, Viajes Importantes, Viva México K’aribe, Oniros Travel, Mayalumm, Pelícanos Tour, Selective Escape, TO Traveler, Solatino Tour, Hola Tours \& Travel, Magitur, Excel Tours, Caribe Viagi, Cenote Azul y Check In Travel, entre otras tantas empresas turísticas de diferentes ramos.

Relacionado con gremio de las agencias de viajes se encuentra la Asociación Mexicana de Agencias de Viajes del Estado de Quintana Roo, creada en 1987, actualmente está constituida por 32 agencias; de los cuales sólo tres son de Playa de Carmen: Best Day, Hola Tours y Olympus Tour.

Lo anterior se puede tomar como referencia para reconocer la importancia que tiene este destino entre los gustos y preferencias de los turistas, tanto nacionales como extranjeros, por lo que el posicionamiento de Playa del Carmen 
depende en gran parte de la calidad del servicio que se ofrezca a un mercado globalizado cada vez más exigente.

Playa del Carmen es considerada como un destino turístico internacional, por lo que resulta importante identificar áreas de oportunidad que permitan seguir colocándolo dentro de mercados turísticos cada vez más exigentes y saturados. Esta investigación pretende apoyar al cumplimiento de ese objetivo, y el producto que se genere de ese análisis estará dirigido al gremio empresarial de las agencias de viajes para mejorar el servicio turístico que brindan, a fin de que este destino, además de ser reconocido por sus atractivos y su planta turística, alcance un elevado nivel de calidad en el servicio y se constituya en un elemento diferenciador dentro el mercado turístico.

\section{Metodología}

El diseño metodológico de la investigación se hizo con un enfoque cuantitativo para identificar la percepción de los clientes, tomando como referencia sus expectativas y necesidades, en relación con el servicio que ofrecen las agencias de viajes de Playa del Carmen.

La presente investigación es de carácter cuantitativo exploratorio, para lo cual se consideró la aplicación del modelo Servqual, herramienta estandarizada que permite identificar el grado de calidad en el servicio que se brinda a los clientes. Este modelo, toma como referencia que la satisfacción del servicio se logra en la medida que el valor o servicio percibido supere o iguale las expectativas de los consumidores, una vez que se haya recibido el servicio.

Para llevar a cabo lo anterior, se establecieron los siguientes objetivos:

- Aplicación del instrumento de recolección de información Servqual con la finalidad de medir la percepción del cliente, considerando cinco niveles de respuestas basados en la escala de Likert.

- Codificación de la información recabada por cada dimensión que establece el modelo Servqual. 
- Interpretación de la percepción de los clientes actuales sobre el servicio que prestan las agencias de viajes en Playa del Carmen tomando como bases sus expectativas.

El cuadro 1 presenta los elementos que se tomaron en cuenta para correlacionar la información.

Cuadro 1. Información correlacionada

\begin{tabular}{lllcc}
\multicolumn{1}{c}{ Variable } & \multicolumn{1}{c}{ Indicadores } & \multicolumn{1}{c}{$\begin{array}{c}\text { Escala Likert } \\
\text { (Niveles de respuesta) }\end{array}$} & $\begin{array}{c}\text { Nivel de } \\
\text { codificación }\end{array}$ & $\begin{array}{c}\text { Escala de } \\
\text { medición }\end{array}$ \\
$\begin{array}{l}\text { Expectativa del } \\
\text { servicio }\end{array}$ & $\begin{array}{l}\text { Dimensión 1: Elementos } \\
\text { tangibles }\end{array}$ & $\begin{array}{l}\text { Totalmente en } \\
\text { desacuerdo (1) }\end{array}$ & -2 & Ordinal \\
& $\begin{array}{l}\text { Dimensión 2: Fiabilidad } \\
\text { Dimensión 3: Capacidad de }\end{array}$ & $\begin{array}{l}\text { En desacuerdo (2) } \\
\text { Indistinto (3) }\end{array}$ & -1 & \\
Percepción del & respuesta. & De acuerdo (4) & 1 & \\
servicio & Dimensión 4: Seguridad & Absolutamente de & 2 & \\
& Dimensión 5: Empatía & acuerdo (5) & & \\
\hline
\end{tabular}

El tipo de muestreo que se aplicó fue no probabilístico a conveniencia. El criterio para participar en el estudio fue haber utilizado los servicios de alguna de las agencias de viajes establecidas en Playa del Carmen, Quintana Roo.

El tamaño de la muestra se definió tomando como base el punto de saturación para una muestra cualitativa propuesto por Bertaux, citado por Mejía (2000), quien establece en 30 el número de casos necesarios para lograr un punto de saturación, y considera que un número mayor a esta cantidad tiende a repetir las características del objeto de estudio. Tomando como referencia lo anterior, se consideró un número mayor a 30, con la finalidad de confirmar las pautas que se fueran presentado en relación al objeto de estudio, procurando así que el resultado de las evidencias fueran más sólidas dentro de lo posible, ya que por la naturaleza del tipo de muestreo seleccionado existe un determinado margen de error. Los cuestionarios se aplicaron entre la segunda semana del mes de septiembre de 2012 hasta la segunda semana de octubre del mismo año. 
Es importante señalar que, a pesar de que todas las brechas propuestas en este modelo son importantes porque ayudan a medir el grado de satisfacción del cliente e identificar sus necesidades, el alcance de este estudio se enfoca únicamente en la brecha 5 , la cual mide el grado de cumplimiento en el servicio entre las expectativas y las percepciones de los consumidores.

Tomando como base el planteamiento anterior, los posibles resultados podrían ser los siguientes según lo considerado por los autores de este modelo :

- Expectativas $>$ Percepción = Bajo grado de calidad

- Expectativas < Percepción = Derroche de calidad

- Expectativas $=$ Percepción $=$ Calidad óptima

El cuestionario estuvo integrado por 22 ítems para conocer las expectativas del consumidor y otros 22 para conocer su percepción. Para identificar el grado de cumplimiento de cada ítem, se consideró la aplicación de la escala respuestas múltiple de Likert, que en este caso se manejó una escala de 5 puntos, lo cual ayudó a la suma de las respuestas de los elementos del cuestionario, para posteriormente realizar la codificación de la información. Los cinco niveles de respuesta fueron los siguientes:

- Totalmente en desacuerdo

- En desacuerdo

- Indistinto

- De acuerdo

- Totalmente de acuerdo

Dentro del instrumento de recolección de datos, se incluyeron dos preguntas demográficas: lugar de procedencia y género del encuestado, considerando estas dos variables como complementarias del estudio. La recolección de datos estuvo a cargo de un grupo de estudiantes del décimo cuatrimestre de la carrera de Licenciado en Gestión y Desarrollo Turístico, de la Universidad Tecnológica de la Riviera Maya. 
Para la codificación, correlación y análisis de los datos obtenidos en las encuestas, se utilizaron hojas de Excel para realizar los gráficos de frecuencia y así comparar en forma visual las percepciones y la expectativas por intervalo de cada una de las cinco dimensiones del modelo Servqual.

Con respecto a la comprobación de la hipótesis que se plantea en esta investigación, se aplicó el análisis de la varianza Anova.

Previo a la recolección de datos, al grupo de encuestadores se les explicó el objetivo de la investigación, así como la importancia de la adecuada aplicación de la herramienta. Los encuestadores se organizaron en diez subgrupos de trabajo de dos integrantes. El objetivo fue realizar cien encuestas durante la segunda semana del mes de septiembre del 2012 hasta la segunda semana del mes de octubre del mismo año.

Para la recolección de datos, los encuestadores se ubicaron en la zona turística de Playa del Carmen, donde se encuentran varias agencias de viajes mayoristas y minoristas como tour operadoras, así como dentro de hoteles, donde se encuentra agencias IMPLANT. Lo anterior con la finalidad de abordar a los clientes al salir de las agencias y encuestarlos sobre el servicio recibido, para obtener una referencia casi inmediata de su percepción.

Una vez recolectados los datos, se realizó un análisis estadístico con la finalidad de contabilizar y promediar los valores cuantitativos asignados por los encuestados para cada ítem, utilizando para ello hojas de Excel, donde también se calcularon sus respectivas medias y la desviación, para confirmar que los valores de las medias fueran representativos en relación con las respuestas, considerando todo el conjunto de cuestionarios aplicados a la muestra. Los promedios permitieron calcular las brechas de cada ítem que conforman la dimensión correspondiente.

Se estableció la siguiente hipótesis:

$\mathrm{H}_{0}$ : No hay diferencia entre las expectativas y las percepciones de los clientes actuales sobre el servicio que brindan las agencias de viajes en Playa del Carmen, lo que refleja un grado de calidad óptimo. 


\section{Resultados}

La muestra final fue de 80 encuestados, debido a que por falta de disponibilidad y de tiempo de algunas personas que se encuestaron, varios cuestionarios quedaron sin completar, o bien la referencia del cliente era de agencias de viajes que no se encontraban en Playa del Carmen; por lo que, considerando que el tipo de muestreo seleccionado fue no probabilístico a conveniencia, eso casos fueron eliminados de la base de datos quedando únicamente 80 casos para estudio, cuyas características demográficas se presentan en el cuadro 2.

Cuadro 2. Características demográficas de la muestra

\begin{tabular}{ccc}
$\begin{array}{c}\text { Total de personas } \\
\text { encuestadas }\end{array}$ & Género & Origen \\
\hline 80 & 41 Mujeres & 44 extranjeros \\
& 39 Hombres & 36 nacionales \\
\hline
\end{tabular}

En cuanto a las agencias de viajes que fueron referencia de los encuestados se encuentran las siguientes: Best Day Travel, Cenote Azul Tours and Travel, To Traveler, Check In Travel, Hola Tours, Solatino Tours and Travel, Olympus Tour Playa del Carmen, Viajes x el Mundo, Excel Tours, Viajes Importantes, Caribe Viaggi, Pelícanos Tours, Oniros Travel, Viajes Mayaluum, Viva México K’aribe, Selective Escape y Magnitour.

Cabe hacer mención que se corroboró la dirección de todas las empresas enlistadas para verificar que efectivamente estuvieran establecidas en Playa del Carmen.

Para analizar e interpretar los datos obtenidos durante el muestreo, se presentan a continuación las gráficas por dimensión, que muestran la correlación que existen entre las expectativas y la percepción de los clientes actuales sobre la calidad del servicio que brindan las agencias de viajes en Playa del Carmen. 
En el siguiente cuadro, se muestra el concentrado de las frecuencias del nivel de respuesta que dieron los encuestados, tanto para las expectativas como para las percepciones, lo que permitirá identificar el grado de cumplimiento por parte de las agencias de viajes en cada una de las cinco dimensiones que considera el modelo Servqual.

Cuadro 3.: Concentrado de frecuencias por nivel de respuesta

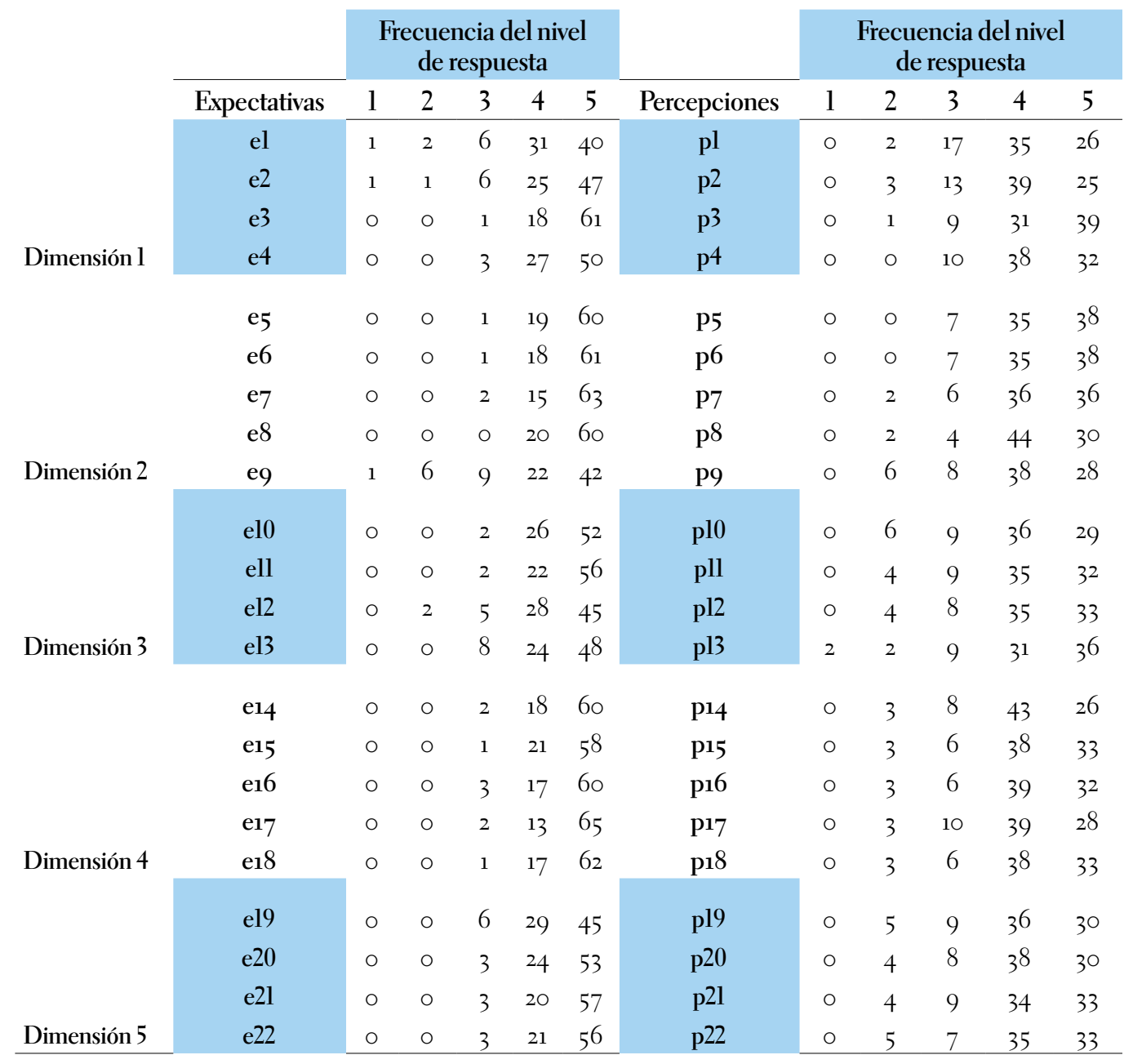




\section{Análisis de la dimensión de elementos tangibles}

Las frecuencias de la figura 1 reflejan el nivel de percepción (rojo) y expectativas (azul) de los clientes y muestran el nivel de satisfacción que se registró dentro de los cuatro ítems que componen la dimensión de elementos tangibles. En cuanto a la percepción que tiene el cliente en relación con la apariencia del personal, instalaciones físicas, equipos y materiales de comunicación, éstas no cumplen con sus expectativas, observándose que la brecha más amplia está en la evaluación del ítem tres, que hace referencia a la apariencia del personal.

La brecha con un margen relativamente más cercano entre las expectativas y la percepción del cliente es la relacionada con la apariencia moderna que tienen los equipos de las agencias de viajes. Sin embargo en ninguno de los ítems la percepción igualó o superó las expectativas, lo que muestra un bajo grado de calidad en el servicio.

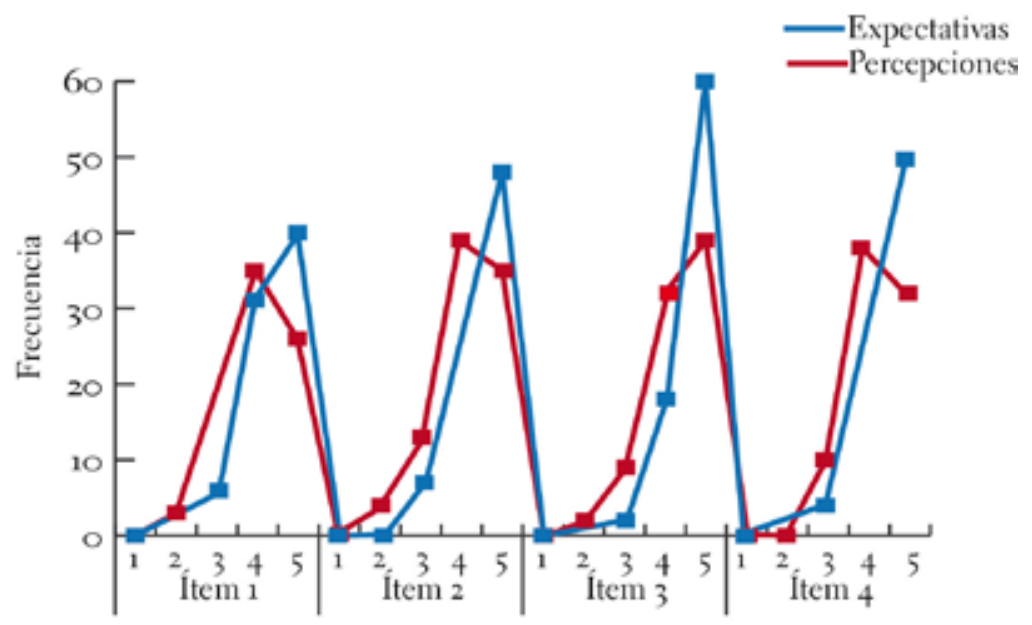

Figura 1. Dimensión 1 / elementos tangibles 


\section{Análisis de la dimensión de fiabilidad}

Esta dimensión está enfocada a evaluar la habilidad con la que cuenta el prestador de servicios para ejecutar de forma fiable y cuidadosa el servicio prometido al cliente. Con base en lo anterior, la figura 2 muestra que la percepción está muy por debajo de las expectativas del cliente. Cabe destacar que en el ítem 9 las expectativas siguen sin cumplirse, pero la brecha es más angosta, por lo que se percibe que el cliente es relativamente consciente que se pueden presentar errores dentro del proceso del servicio, pero también demanda pericia en la resolución de cualquier problema que se pudiera presentar, así como el cumplimiento de lo prometido en tiempo y forma.

Por lo que se concluye en el análisis de esta dimensión que las expectativas, al no ser igualadas o superadas por la percepción del cliente, reflejan un bajo grado de satisfacción en el servicio en el aspecto de fiabilidad, considerando su amplitud entre las brechas.

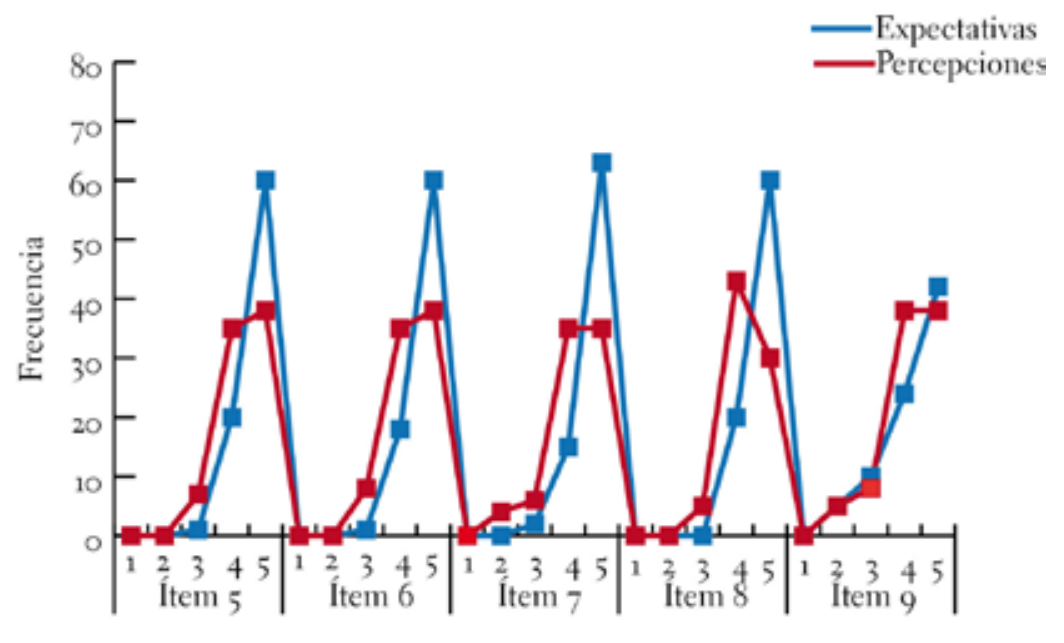

Figura 2. Dimensión 2 / fiabilidad 


\section{Análisis de la dimensión de capacidad de respuesta}

Con respecto a la dimensión en la que se evalúa la capacidad de respuesta que tiene el prestador de servicios en cuanto a disposición y voluntad por ayudar y orientar al cliente, la figura 3 reflejan una brecha importante entre las expectativas, que son altas, en relación con la percepción que tiene el cliente sobre del servicio prestado, mostrando una deficiencia en la rapidez, disponibilidad y baja capacidad de respuesta, al no comunicar de manera oportuna y completa la información que pudiera ser necesaria durante todo el proceso de servicio, considerando que este finaliza hasta que el cliente haya consumido el producto turístico que comercializa la agencia de viajes.

Por lo anterior, se puede decir que existe una percepción del servicio de baja calidad al no tener el nivel de capacidad de respuesta esperado por el cliente por parte de los colaboradores de estas empresas objeto de estudio.

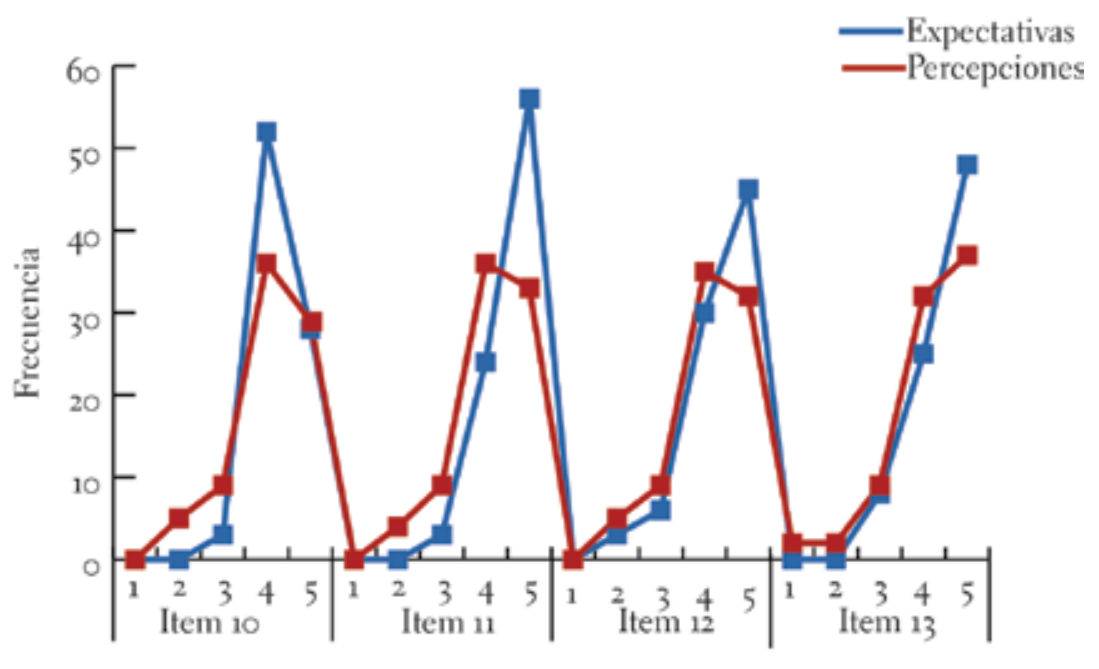

Figura 3. Dimensión 3 capacidad de respuesta 


\section{Análisis de la dimensión de seguridad}

La dimensión de seguridad evalúa el conocimiento y atención de los prestadores de servicios, considerando además sus habilidades para inspirar credibilidad y confianza a los clientes. La figura 4 muestra que el cliente tiene altas expectativas y esto se refleja de manera equitativa en el nivel de frecuencia correspondiente de los cinco ítems que permiten evaluar esta dimensión.

Es importante hacer mención que el nivel de percepción se muestra muy semejante en cuando a su frecuencia, el cual es casi la mitad de las expectativas; evidenciándose así una brecha considerable que permite determinar que, al no igualarse o superarse las expectativas de los clientes, éstos perciben un bajo grado de calidad en el servicio por parte de los empleados que laboran en las agencias de viajes en Playa del Carmen, en cuanto a seguridad se refiere.

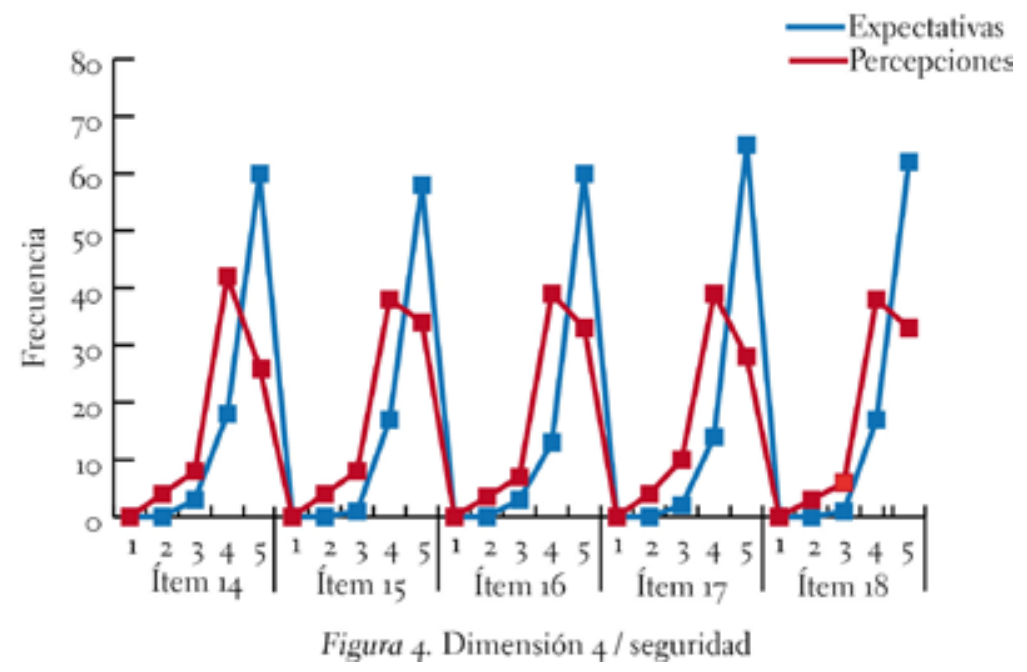




\section{Análisis de la dimensión de empatía}

Esta dimensión evalúa el grado de cumplimiento en la atención individualizada que ofrecen las empresas a sus clientes. En este sentido, la figura 5 muestra que las expectativas son altas, principalmente al exigir el cliente un mayor interés y comprensión de sus necesidades de parte de los prestadores de servicios; lo cual se refleja principalmente en el nivel de respuesta captado en los ítems 21 y 22.

La brecha más angosta se presenta en el ítem 19 que evalúa la conveniencia en cuanto a horarios de trabajo para la atención al público. A pesar de que también aquí las expectativas sobrepasan la percepción, se puede considerar que hay una comprensión relativa del cliente con respecto a los horarios de servicio.

Analizando lo anterior se puede concluir que en esta dimensión el cliente percibe un bajo grado de calidad en el servicio al no cumplir con sus expectativas.

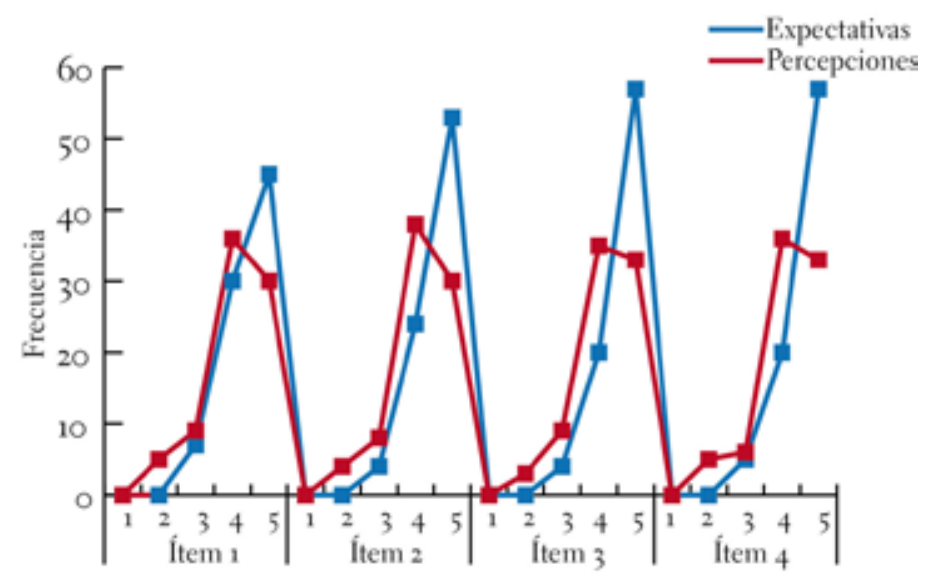

Figura 5. Dimensión 5/empatía 
Se realizó una comparación de respuestas por sexo, como lo muestra la la figura 6. El resultado indica que en cuanto a expectativas, ambos sexos tienen respuestas muy similares, la mayoría entre 4 y 5; mujeres y hombres están muy de acuerdo con el tipo de servicio esperado. Sin embargo, respecto a la percepción, la mayoría de las respuestas del sexo masculino es de 4; es decir, el sexo masculino califica más bajo el servicio recibido, lo que evidencia que este género percibe más las fallas. Mientras tanto, el sexo femenino lo califica mejor, dando más frecuentemente la calificación de 5; está más de acuerdo con el servicio recibido.

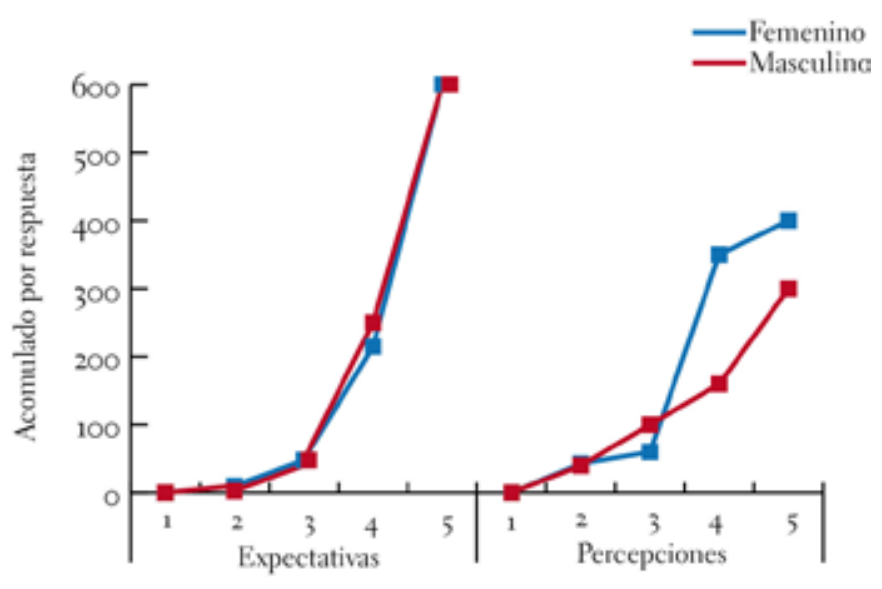

Figura 6. Cuadro de expectativas y percepciones por sexo

\section{Discusión}

Es importante hacer notar que en ninguna de las cinco dimensiones que componen el modelo Servqual las percepciones igualaron o superaron las expectativas, por lo que los resultados permiten rechazar la hipótesis de esta investigación, en la que se plantea que no hay diferencia entre las expectativas y las percepciones de los clientes sobre el servicio que brindan las agencias de viajes en Playa del Carmen, lo que reflejaría un grado de calidad óptimo. Los datos analizados mostraron que las percepciones de los clientes actuales sobre el servicio que ofrecen estas empresas en el destino son inferiores a sus expectativas, lo que refleja un bajo grado de calidad en el servicio. 
Considerando lo anterior, se puede identificar que existe una problemática importante que afecta tanto al destino como al sector que representan las agencias de viajes de Playa del Carmen, al ser evaluado su servicio con un grado de deficiencia que no permite satisfacer las expectativas de sus clientes. Por lo tanto resulta importante que se consideren estrategias que permitan mejorar el servicio.

Las agencias de viajes, como representantes e intermediarias de productos turístico que ofertan otras empresas del sector (hoteles, líneas aéreas, restaurantes, parques recreativos, arrendadoras de autos, cruceros, empresas de transporte terrestre, etc), deberían tener un gran compromiso en la calidad de su propio servicio y garantizar también el que ofrecerán sus proveedores, los cuales en algún momento del proceso darán servicio directo al cliente que los contrató con la agencia de viajes, influenciando también de manera directa o indirecta en las expectativas del cliente; lo que impactará en el proceso de compra y esto puede ser positivo o negativo, dependiendo de la experiencia que haya vivido del cliente.

Considerando el análisis de los resultados, es de resaltar la información obtenida en la dimensión 2, la cual evalúa la fiabilidad; donde cuatro de los cinco ítems que componen esta dimensión, muestran una brecha entre la percepción y la expectativa muy amplia y relativamente igual en longitud entre estos ítems. Lo anterior llevaría a pensar que el cliente espera que el prestador de servicio tenga una gran habilidad para ejecutar el servicio de manera fiable y cuidadosa; lo que implicaría la anticipación de detección de necesidades de manera eficiente, con la finalidad de concretar en tiempo y forma lo solicitado, procurando minimizar o evitar errores en el proceso. Pero al respecto, el cliente percibe que esto se cumple en un grado muy bajo, lo que hace que la brecha sea amplia.

En la fiabilidad interviene en elemento muy importante, que es el tiempo, el cual se puede optimizar si se comprenden las funciones y la importancia de las tareas a desempeñar, considerando ante todo la detección correcta de necesidades. 
Existen teorías como la de Jan Carlzon, llamada Momentos de la verdad, la cual refiere que bastan sólo 15 segundos en los cuales los empleados de una organización ponen a prueba a toda la empresa al momento del primer contacto con sus clientes; y la imagen, la pericia en las actividades que ejecutan los empleados para entregar el servicio, así como la empatía, reflejan seguridad, credibilidad y confianza al cliente. Por lo que en ese sentido se percibe que los resultados de las cinco dimensiones pueden estar correlacionadas, y el grado de evaluación asignado por el cliente, en un determinado momento, se refleja en las otras dimensiones, pues aunque hubo brechas angostas reflejadas en algunos ítems, se identificó una constante en la amplitud de las brechas en todas las dimensiones.

Como se mencionó, la dimensión de la fiabilidad, que hace referencia al correcto funcionamiento del servicio, mostró brechas muy amplias en los ítems evaluados, seguida de la dimensión de seguridad que evalúa la confianza y credibilidad que inspiran los prestadores de servicios a su clientes, que ha sido el siguiente punto crítico que mostró un grado importante de incumplimiento en la calidad del servicio percibido por el cliente. En seguida se encuentra la dimensión de la empatía que esta orientada a evaluar la comprensión de las necesidades de los clientes; seguida por la capacidad de respuesta y, por último, las brechas que muestran relativamente menos amplitud entre las expectativas y las percepciones es en la dimensión que evalúa los elementos tangibles, donde sobresale la amplitud del ítem 3 que hace referencia a la apariencia de los empleados.

Otro punto relevante para analizar son los resultados de la variable demográfica relacionada con el género de los encuestados; donde se refleja que tanto el hombre como la mujer tienen un grado de exigencia semejante en relación con sus expectativas sobre el servicio. Pero por el lado de la percepción, el género masculino califica de manera más reservada o moderada el nivel de servicio recibido; mientras que la mujer otorga una mejor calificación al evaluar el servicio que le fue ofrecido.

Lo anterior puede sugerir que los hombres, además de ser exigentes con sus expectativas, también son más congruentes, en lo que podrían influir varios 
factores; por ejemplo, el tener la responsabilidad de todo lo que implica un viaje o la compra de un servicio; considerando los cánones o patrones que marcan las diferentes sociedades, que ubican al hombre como proveedor, procurador y protector, llevándolo a mostrar una exigencia para cumplir con lo anterior y ser más crítico al respecto. Pudiendo no ser así para la mujer, que a pesar de que hoy en día se desenvuelve de manera más independiente en todo sentido, su naturaleza puede influir, siendo más flexible y tolerante con el servicio recibido; esto con respecto a los resultados que muestran que la mujer esta más de acuerdo con el servicio que recibe por parte de estas empresas; sin embargo, sólo quedará a manera de apreciación, ya que podría ser un tema que complemente este estudio en un futuro.

Dentro de su carácter de intermediara, las agencias de viajes se convierten, en la mayoría de los casos, en el primer contacto para el cliente que busca un producto que satisfaga sus necesidades, confiando en el conocimiento y profesionalismo del personal que labora en estas empresas por lo que el garantizar un alto grado de calidad sería un elemento diferenciador de la competencia, concretando en una fidelización que beneficie a los diferentes actores del turismo que intervienen en el proceso.

La técnica de inferencia estadística para la prueba de hipótesis se basó en la diferencia de medias, considerando dos muestras dependientes. Los datos obtenidos se comparan directamente entre sí usando la diferencia de sus valores numéricos, llamada diferencia pareada. La cual coincide con la relación indicada en el modelo Servqual:

Expectativas - Percepción $=$ Calidad en el servicio (grado de cumpliento en el servicio)

La hipótesis nula, $\mathrm{H}_{0}$, indica que no hay diferencia entre expectativas y percepción, es decir:

$$
H_{0}=0
$$


Con los resultados codificados se obtuvo el promedio de la diferencia pareada de las muestras así como su desviación estándar. El estadístico de prueba $t$ de student utilizado fue:

$$
\begin{array}{lll}
t^{*}=\frac{\bar{d}-\mu_{d}}{s_{d} / \sqrt{n}} & & \bar{d}=0.40965909 \\
t^{*}=3.98698253 & \text { donde: } & \mu_{d}=0 \\
& s_{d}=0.91901639 \\
& n=80
\end{array}
$$

Probado a un nivel de significancia $=0.05 \mathrm{y} g \mathrm{l}=79$, el resultado de la probabilidad asociada a t* fue de aproximadamente 0 , concluyendo al nivel de significancia de 0.05 se rechaza la hipótesis nula, es decir con los datos obtenidos se sustenta que sí existe diferencia entre las expectativas y las percepciones de los clientes al recibir un servicio.

\section{Conclusiones}

Esta investigación arrojó resultados que mostraron que los clientes de las agencias viajes de Playa del Carmen reciben un bajo grado de calidad en el servicio, lo que implica una insatisfacción en sus necesidades. El hallazgo anterior llevó a rechazar la hipótesis que se planteó en presente trabajo, la cual establecía que no existía diferencia entre la percepción y la expectativa del cliente lo que reflejaría un nivel óptimo de calidad en el servicio.

Lo anterior se pudo identificar con la aplicación del modelo Servqual, propuesto por Parasuraman, Zeithaml y Berry, por medio del cual se obtuvo información que mostró que las expectativas de los clientes de estas empresas no se cumplen en ninguna de las cinco dimensiones que conforman el modelo (tangibilidad, fiabilidad, capacidad de respuesta, seguridad y empatía). Por lo anterior, el área de oportunidad en el que las agencias de viajes pudieran orientar sus esfuerzos para satisfacer las necesidades de sus clientes, está enfocada en elevar el grado de calidad del servicio que ofrecen, que permita superar las expectativas de los clientes. 
Hablar de la calidad en el servicio, implica monitorear el grado de satisfacción que percibe el cliente, en relación con sus expectativas. Para lograr lo anterior, las empresas deben establecer objetivos de calidad, que permitan el cumplimiento de los requisitos, así como su aseguramiento, proporcionando confianza dentro de todo el proceso de servicio. Lo anterior puede ser la base para dar cumplimiento a las expectativas de los clientes, llegando a su satisfacción y fidelización .

Llevar a cabo la mejora continua es otra acción igual de importante y que también forma parte de la gestión de la calidad. Este tipo de acciones permiten identificar áreas de oportunidad para proponer estratégicas que pudieran ser tomadas en cuenta en determinado momento, con la finalidad de dar cumplimiento a la mejora continua para seguir dentro del contexto de la competitividad. Lo anterior con base en que la calidad en el servicio ya no debería ser una opción, ya que hoy en día se puede considerar un elemento diferenciador y no complementario.

El análisis y los resultados de este estudio puede ser referencia de nuevas investigaciones que permitan seguir contribuyendo con información sobre el comportamiento del sector. Al respecto se considera, en una segunda etapa, complementar este estudio, generando una investigación orientada a identificar la relación que existe entre el nivel de satisfacción del cliente y el grado de profesionalización y formación académica del recurso humano que labora en las agencias de viajes de Playa del Carmen.

\section{Literatura consultada}

Chan, N. (2005). Circuitos turísticos. Programación y cotización. Buenos Aires: Turísticas.

González, M., Chamorro Mera, A., y Rubio Lacoba, S. (2007). Introducción a la gestión de la calidad. Madrid: Delta Publicaciones.

Juran, J. (1996). Juran y la calidad por el diseño. Madrid: Ediciones Díaz de Santos. 
Mejía Navarrete, J. (2000). El muestreo en la investigación cualitativa. Investigaciones Sociales, 165-180.

Rivera, J., López-Rúa, M. (2007) Dirección de marketing. Fundamentos y aplicaciones. Madrid: Editorial EsIC

\section{Referencias electrónicas}

Asociación Mexicana de Agencias de Viajes de Quintana Roo, A. C. http:// www.amavqroo.mx/felicidades-amav-q-roo/

Ayuntamiento de Solidaridad (2008). http://tecnica.qroo.gob.mx/portal/documentos/Perfiles_Municipales/SOLIDARIDAD.pdf

Caribe mexicano. http://www.caribemexicano.gob.mx/

Federación Turística de Quintana Roo. http://www.fedeturquintanaroo.com/ delegacion_riviera_maya.html

Ofertas de viajes México. http://www.ofertasdeviajesmexico.com/es/cont/socios_filiares/Directorio_Filial_Quintana_Roo.php

Secretaría de Turismo. http://www.sectur.gob.mx/work/models/sectur/Resource/14944/LEY_GENERAL_DE_TURISMO.pdf

Sistema Integral de Información de Mercados Turísticos. http://www.siimt.com United Federation of Travel Agents' Associations. http://www.uftaa.org 